\title{
Trapezoid-Capitate Joint
}

National Cancer Institute

\section{Source}

National Cancer Institute. Trapezoid-Capitate Joint. NCI Thesaurus. Code C142327.

The articulation of the trapezoid and capitate bones in the wrist. 